\title{
The Non-Muscle Myosin Heavy Chain 9 Gene (MYH9) Is Not Associated with Lupus Nephritis in African Americans
}

\author{
Barry I. Freedman ${ }^{\text {a J Jeffrey C. Edberg }}{ }^{\text {b }}$ Mary E. Comeau ${ }^{a}$ Mariana Murea ${ }^{a}$ \\ Donald W. Bowden ${ }^{a}$ Jasmin Divers ${ }^{a}$ Graciela S. Alarcón ${ }^{b}$ Elizabeth E. Brown ${ }^{b}$

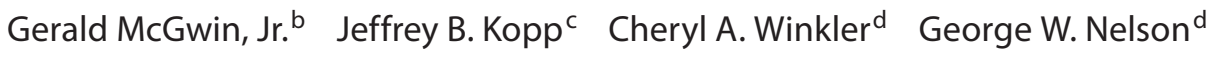 \\ Gabor Illei ${ }^{\mathrm{e}}$ Michelle Petri ${ }^{f}$ Rosalind Ramsey-Goldman ${ }^{g}$ John D. Reveille ${ }^{\mathrm{h}}$ \\ Luis M. Vilái Carl D. Langefeld ${ }^{\mathrm{a}}$ Robert P. Kimberly ${ }^{\mathrm{b}}$ for the PROFILE Study Group \\ aWake Forest University School of Medicine, Winston-Salem, N.C., b University of Alabama at Birmingham, \\ Birmingham, Ala., ' Kidney Disease Section, National Institute of Diabetes and Digestive and Kidney Diseases, NIH,

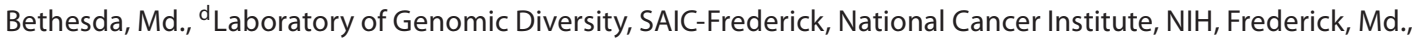 \\ 'Gene Therapy and Therapeutics Branch, National Institute of Dental and Craniofacial Research, NIH, Bethesda, Md., \\ fJohns Hopkins University School of Medicine, Baltimore, Md., 9 Northwestern University Feinstein School of \\ Medicine, Chicago, III., h University of Texas Health Science Center at Houston, Houston, Tex., and 'University of \\ Puerto Rico Medical Sciences Campus, San Juan, P.R., USA
}

\section{Key Words}

African Americans · Genetics • Lupus nephritis • Kidney • $\mathrm{MYH9} \cdot$ Systemic lupus erythematosus

\begin{abstract}
Background: African Americans (AA) disproportionately develop lupus nephritis (LN) relative to European Americans and familial clustering supports causative genes. Since $\mathrm{MYH} 9$ underlies approximately $40 \%$ of end-stage renal disease (ESRD) in AA, we tested for genetic association with LN. Methods: Seven MYH9 single nucleotide polymorphisms (SNPs) and the E1 risk haplotype were tested for association with LN in three cohorts of AA. Results: A preliminary analysis revealed that the $\mathrm{MYH} 9 \mathrm{E} 1$ risk haplotype was associated with ESRD in 25 cases with presumed systemic lupus erythematosus (SLE)-associated ESRD, compared to 735 non-SLE controls (odds ratio 3.1; $p=0.010$ recessive). Replication analyses were performed in 583 AA with SLE in the PROFILE cohort (318 with LN; 265 with SLE but without nephropathy)
\end{abstract}

and 60 AA from the NIH (39 with LN; 21 with SLE but without nephropathy). Analysis of the NIH and larger PROFILE cohorts, as well as a combined analysis, did not support this association. Conclusions: These results suggest that $A A$ with ESRD and coincident SLE who were recruited from dialysis clinics more likely have kidney diseases in the MYH9-associated spectrum of focal segmental glomerulosclerosis. PROFILE and $\mathrm{NIH}$ participants, recruited from rheumatology practices, demonstrate that $\mathrm{MYH} 9$ does not contribute substantially to the development of LN in AA.

Copyright $\odot 2010$ S. Karger AG, Basel

\section{Introduction}

Relative to European Americans (EA), African Americans (AA) are at markedly increased risk for developing kidney disease associated with systemic lupus erythematosus (SLE), type 2 diabetes mellitus, focal segmental glomerulosclerosis (FSGS), human immunodeficiency virus

\section{KARGER}

() 2010 S. Karger AG, Basel

Fax +41613061234 E-Mail karger@karger.ch www.karger.com www.karger.com/ajn
Barry I. Freedman, MD

Section on Nephrology, Wake Forest University School of Medicine

Medical Center Boulevard, Winston-Salem, NC 27157-1053 (USA)

Tel. +1 336716 6192, Fax +1 3367164318

E-Mail bfreedma@wfubmc.edu 
infection (HIV-associated nephropathy; HIVAN) and focal global glomerulosclerosis (FGGS, historically labeled 'hypertension-associated nephropathy'). After initial detection using mapping by admixture linkage disequilibrium, multiple regions within $M Y H 9$ have shown strong and replicated associations with FSGS, FGGS and HIVAN in AA with end-stage renal disease (ESRD), with odds ratios (OR) between 3 and 5 [1-3]. MYH9 demonstrated a weaker association in type 2 diabetes-associated ESRD in AA [4]. As such, MYH9 contributes to approximately $40-45 \%$ of all ESRD in the AA population. Risk variants in $\mathrm{MYH} 9$ are present in $60 \%$ of AA and $4 \%$ of EA (approximately $36 \%$ of AA are E1 risk haplotype homozygotes, compared to less than $1 \%$ of EA), explaining much of the ethnic variation in incidence rates of kidney disease [1-3].

SLE affects AA significantly more often than EA [5], and AA have more severe renal involvement [6]. Only a subset of SLE patients ultimately develops lupus nephritis (LN) and the factors underlying this phenomenon remain elusive. Familial aggregation of LN has been observed and close relatives of patients with LN often have disparate (e.g. non-lupus-related) etiologies of nephropathy [7]. This finding suggested the existence of overarching renal failure susceptibility genes, nephropathy genes not necessarily specific for LN $[8,9]$. It is also unclear whether genes underlying severe forms of LN (e.g. ESRD) contribute to milder forms of $\mathrm{LN}$.

To determine whether the MYH9 gene underlies susceptibility to LN, seven MYH9 single nucleotide polymorphisms (SNPs) strongly associated with ESRD were tested for association with LN in $668 \mathrm{AA}$ patients with SLE recruited in the PROFILE and NIH cohorts and at the Wake Forest University School of Medicine(WFUSM). In addition, 735 AA control subjects without SLE were genotyped.

\section{Patients and Methods}

\section{Study Populations}

25 AA with ESRD clinically attributed to LN (listed on the CMS 2728 diagnosis code form) and 735 AA controls denying a personal history of SLE or kidney disease were recruited at the WFUSM. The PROFILE cohort consisted of 583 AA with SLE, as previously reported [10]. PROFILE participants included in this investigation consisted of 583 AA SLE patients meeting at least 4 of 11 American College of Rheumatology (ACR) criteria for SLE. Of these, 318 had LN (cases) and 265 SLE patients did not have this clinical manifestation (controls) as defined by one or more renal function evaluations. The NIH cohort consists of 60 subjects with SLE (39 with LN) and $96.7 \%$ of subjects had longitudinal data on renal function (minimum 2-year follow-up after the diagnosis of SLE). All subjects gave informed consent for genetic studies, under protocols approved by the relevant Institutional Review Board.

SNP Selection and Genotyping

Seven SNPs in MYH9 were chosen for genotyping, based on strong association with other forms of ESRD in previous reports containing AA with FSGS, FGGS, HIVAN and diabetes-associated ESRD [1, 3]. Tested SNPs included rs11912763, rs4821480, rs2032487, rs4821481, rs5750250, rs3752462, and rs5756152 (see table 2). The strongly associated MYH9 E1 risk haplotype containing rs4821480, rs2032487, rs4821481 and rs3752462 was also evaluated [1]. SNP genotyping was performed on a TaqMan Genotyping System (Applied Biosystems, Carlsbad, Calif., USA).

Seventy di-allelic ancestry informative markers (AIMs) were genotyped to provide individual admixture proportion estimates used to control for population substructure in the association tests from the WFUSM samples (AIM genotypes were not available in the NIH samples). A principal components analysis was performed in the PROFILE participants using genome-wide association data to account for population structure. AA case and control DNA samples for AIMs were genotyped using either Illumina Inc.'s Custom Genotyping Services (San Diego, Calif., USA) or using the Sequenom Mass Array (San Diego, Calif., USA). 39 unrelated EA controls were recruited, as described for the AA controls, and DNA was obtained from 44 Yoruban Nigerians (YRI) from the National Institute of General Medicine Sciences (NIGMS) Human Variation Collection (Coriell Repositories, Camden, N.J., USA).

\section{Statistical Analyses}

Each SNP was tested for departures from Hardy-Weinberg equilibrium (HWE) expectations via a $\chi^{2}$ goodness-of-fit test [11]. Linkage disequilibrium was estimated using the classic $\mathrm{D}^{\prime}$ and $\mathrm{r}^{2}$ statistics as implemented in Dprime (http://www.phs.wfubmc. edu/public_bios/sec_gene/downloads.cfm) and Haploview 3.32 [12]. All tests of association were adjusted for age and gender and all analyses limited to WFUSM samples were ancestry-adjusted. The individual admixture proportions for the Wake Forest University Health Sciences samples were estimated via the expectation maximization (E-M) algorithm implemented in the software FRAPPE $[13,14]$. Analyses using the PROFILE samples were adjusted for ancestry using principal components, however those from the NIH were not (as these data were unavailable). The primary inference is based on the recessive model of the E1 MYH9 haplotype, consistent with previously published and replicated associations of that specific model. Other tests are only provided for additional information and completeness. Specifically, to test for an association between each SNP and LN we computed the overall genotypic test of association and the three a priori genetic models (dominant, additive and recessive). We tested for departures from additivity and computed the allelic and four-marker E1 haplotype. These tests were computed using SNPGWA and Dandelion (http:// www.phs.wfubmc.edu/public_bios/sec_gene/downloads.cfm) both programs use the E-M algorithm for haplotype estimation. Both the large-sample and permutation tests were computed to estimate statistical significance. Finally, we performed a combined analysis in all PROFILE and NIDDK samples genotyped for 5 common SNPs; this analysis was not ancestry-adjusted due to lack of AIMs or principal components in the NIH sample. 
Table 1. Demographic data in patients with SLE

\begin{tabular}{llllll}
\hline Variable & $\begin{array}{l}\text { Profile LN } \\
\text { cases } \\
(\mathrm{n}=318)\end{array}$ & $\begin{array}{l}\text { Profile SLE } \\
\text { controls without } \\
\text { LN }(\mathrm{n}=265)\end{array}$ & $\begin{array}{l}\text { WFUSM } \\
\text { SLE-ESRD cases } \\
(\mathrm{n}=25)\end{array}$ & $\begin{array}{l}\text { NIH LN } \\
\text { cases } \\
(\mathrm{n}=39)\end{array}$ & $\begin{array}{l}\text { NIH SLE } \\
\text { controls without LN } \\
(\mathrm{n}=21)\end{array}$ \\
\hline Female sex, \% & 93 & 94 & 88 & 89 & 85 \\
Age, years & $29.7(29) \pm 11.3$ & $35.3(34) \pm 11.5$ & $42.3(45) \pm 12.6$ & $26.5 \pm 9.7$ & $33.5 \pm 11.8$ \\
Number of ACR ${ }^{1}$ criteria $^{2}$ & $6.9(7) \pm 1.8$ & $6.9(7) \pm 1.8$ & unavailable & unavailable & unavailable \\
Serum creatinine $\geq 2.5 \mathrm{mg} / \mathrm{dl}, \%^{3}$ & 9.6 & 1.1 & 100 & 4.9 \\
SLE duration, years $^{3}$ & $9.5(8.5) \pm 6.8$ & $7.3(6.4) \pm 5.0$ & unavailable & $14.5 \pm 9.4$ & $13.7 \pm 6.4$ \\
\hline
\end{tabular}

Values are mean (median) \pm SD unless noted otherwise.

${ }^{1}$ American College of Rheumatology. ${ }^{2}$ Excluding renal involvement. ${ }^{3}$ Based on a subset with available data.

\section{Results}

Table 1 contains demographic data in all cases and controls. Twenty-five unrelated AA cases with SLE preceding ESRD were recruited at the WFUSM, along with 735 controls lacking SLE. Available medical records, renal histopathology and laboratory data were reviewed in these 25 cases with ESRD and none had co-morbidities other than SLE felt likely to cause kidney disease (e.g. none had diabetes mellitus, HIV infection, hepatitis B or hepatitis $C$ viral infection). ESRD cases were $88 \%$ female with mean (SD) age at ESRD $42.3 \pm 12.6$ years and 7 reportedly had renal-biopsy evidence of $\mathrm{LN}$ ( 4 of the renal biopsy reports were available; all revealed diffuse proliferative glomerulonephritis, 1 on a background of membranous nephropathy). Medical records in the 18 cases lacking kidney biopsies revealed 4 cases had anti-doublestranded DNA titers $\geq 1 / 160$ with proteinuria exceeding $5.2 \mathrm{~g} /$ day (maximum $11.6 \mathrm{~g} /$ day). The causes of ESRD listed in the US Renal Data Systems may not always be accurate $[15,16]$. In an attempt to clarify this question, we contacted surviving SLE patients with ESRD recruited at WFUSM, their families and physicians. The nephrologists caring for these 25 ESRD patients confirmed the clinical diagnosis of LN and we could not detect risk factors for non-SLE nephropathies in these cases.

The second association analysis was performed in the 583 AA PROFILE participants. 93\% of PROFILE LN cases and 94\% of SLE controls were female (table 1). Age at SLE onset was $29.7 \pm 11.3$ years in LN cases and $35.3 \pm$ 11.5 years in non-nephropathy controls $(\mathrm{p}<0.0001)$. Mean (median) \pm SD SLE duration and number of ACR SLE diagnostic criteria in cases versus controls, respectively, were $9.5(8.5) \pm 6.8$ vs. $7.3(6.4) \pm 5.0$ years $(\mathrm{p}=$ $0.001)$, and $6.9(7) \pm 1.8$ vs. $6.9(7) \pm 1.8(\mathrm{p}=0.96)$, re- spectively. In PROFILE, $71.2 \%$ of $\mathrm{LN}$ cases and $66 \%$ of SLE controls, respectively, had longitudinal measures of renal parameters; $28.8 \%$ of cases and $34 \%$ of controls had a single measure.

The third association analysis was performed using 60 lupus cases at the NIH Clinical Center, of whom 39 manifested LN and 21 did not after a follow-up interval of 14.5 \pm 9.4 and $13.7 \pm 6.4$ years, respectively. Among LN cases, $89 \%$ were female; among SLE controls, $85 \%$ were female. Mean ages at entry into the study cohort were 26.5 \pm 9.7 and $33.5 \pm 11.8$ years, respectively. All SNPs met HWE expectations in the WFUSM, NIH and PROFILE cases and controls.

Tables 2 and 3, respectively, contain association results with the seven MYH9 single SNPs and the E1 haplotype in Wake Forest SLE-ESRD cases and non-SLE controls. Despite the small sample, the a priori recessive model form of the E1 haplotype was associated with SLE-ESRD (OR 3.09; 95\% CI 1.31-7.27; p = 0.010), as were 4 of 7 individual SNPs (OR 2.37-4.31; p values ranged from 0.022 to 0.05$)$.

Tables 4 and 5, respectively, contain results of the single SNP and E1 haplotype replication analyses from the 300 PROFILE LN cases and 246 SLE non-nephropathy controls with data on principal components. No evidence of association was detected between MYH9 single SNPs (table 4) or the E1 haplotype (OR 0.79; 95\% CI 0.55-1.15; $\mathrm{p}=0.22$ recessive). In addition, analyses adjusting solely for age and gender in the full PROFILE sample of $318 \mathrm{LN}$ cases and 265 SLE controls had similar results (data not shown). These results suggested that heterogeneity existed between the PROFILE LN cases and the Wake Forest SLE-ESRD cases, or that the different types of control groups contributed to results (non-SLE controls in Wake Forest and SLE non-nephropathy controls in PROFILE). 
Table 2. MYH9 association analysis results in the Wake Forest sample (25 SLE-ESRD cases vs. 735 non-SLE controls)

\begin{tabular}{|c|c|c|c|c|c|c|c|c|}
\hline \multirow[t]{2}{*}{ Marker } & \multirow{2}{*}{$\begin{array}{l}\text { Risk } \\
\text { allele }\end{array}$} & \multicolumn{2}{|c|}{ Minor allele frequency } & \multicolumn{2}{|c|}{ HWE p value } & \multicolumn{3}{|c|}{ Recessive test } \\
\hline & & cases & controls & cases & controls & OR & $95 \%$ CI & $\mathrm{p}$ value \\
\hline rs11912763 & A & 0.28 & 0.19 & 0.30 & 0.47 & 4.31 & $1.14,16.34$ & 0.0319 \\
\hline rs4821480 & G & 0.76 & 0.61 & 0.07 & 0.10 & 2.60 & $1.06,6.37$ & 0.0361 \\
\hline rs2032487 & $\mathrm{C}$ & 0.75 & 0.62 & 0.11 & 0.11 & 2.37 & $1.00,5.62$ & 0.0496 \\
\hline rs4821481 & $\mathrm{C}$ & 0.77 & 0.61 & 0.06 & 0.12 & 2.82 & $1.16,6.84$ & 0.0217 \\
\hline rs5750250 & A & 0.35 & 0.49 & 1.00 & 0.88 & 0.56 & $0.16,1.93$ & 0.3577 \\
\hline rs3752462 & $\mathrm{T}$ & 0.85 & 0.73 & 0.43 & 0.25 & 2.06 & $0.79,5.35$ & 0.1398 \\
\hline rs5756152 & A & 0.35 & 0.25 & 1.00 & 1.00 & 1.78 & $0.50,6.40$ & 0.3757 \\
\hline
\end{tabular}

Table 3. Haplotypes of MYH9 with association of LN in the Wake Forest sample: 25 SLE-ESRD cases vs. 735 non-SLE controls

\begin{tabular}{llllllll}
\hline \multirow{2}{*}{$\begin{array}{l}\text { E1 } \\
\text { haplotype }\end{array}$} & $\begin{array}{l}\text { Genetic } \\
\text { model }\end{array}$ & \multicolumn{2}{l}{ Haplotype frequency } & & \multicolumn{2}{l}{ Recessive test } & \\
& & cases & controls & & OR & $95 \%$ CI & p value \\
\hline GCCT & dominant & 0.76 & 0.57 & & 1.52 & $0.44,5.22$ & 0.5090 \\
& additive & & & 2.05 & $1.06,3.98$ & 0.0332 \\
& recessive & & & 3.09 & $1.31,7.27$ & 0.0099 \\
\hline
\end{tabular}

Table 4. MYH9 association analysis in PROFILE participants with principal component data (300 LN cases vs. 246 SLE non-nephropathy controls)

\begin{tabular}{|c|c|c|c|c|c|c|c|c|}
\hline \multirow[t]{2}{*}{ Marker } & \multirow{2}{*}{$\begin{array}{l}\text { Risk } \\
\text { allele }\end{array}$} & \multicolumn{2}{|c|}{ Minor allele frequency } & \multicolumn{2}{|c|}{ HWE p value } & \multicolumn{3}{|c|}{ Recessive test } \\
\hline & & cases & controls & cases & controls & OR & $95 \%$ CI & $\mathrm{p}$ value \\
\hline rs11912763 & A & 0.22 & 0.20 & 0.50 & 0.84 & 1.00 & $0.40,2.51$ & 0.9976 \\
\hline rs 4821480 & G & 0.66 & 0.70 & 0.09 & 0.02 & 0.76 & $0.53,1.09$ & 0.1327 \\
\hline rs2032487 & $\mathrm{C}$ & 0.63 & 0.68 & 1.00 & 0.66 & 0.74 & $0.52,1.06$ & 0.0992 \\
\hline rs4821481 & $\mathrm{C}$ & 0.62 & 0.66 & 0.46 & 1.00 & 0.79 & $0.55,1.14$ & 0.2075 \\
\hline rs5750250 & $\mathrm{A}$ & 0.47 & 0.46 & 0.73 & 0.79 & 1.11 & $0.72,1.72$ & 0.6340 \\
\hline rs3752462 & $\mathrm{T}$ & 0.71 & 0.71 & 0.68 & 0.64 & 0.87 & $0.61,1.24$ & 0.4480 \\
\hline rs5756152 & A & 0.29 & 0.25 & 0.26 & 1.00 & 1.07 & $0.52,2.22$ & 0.8527 \\
\hline
\end{tabular}

PROFILE controls are felt to be the optimal sample for detecting nephropathy genes, because all had SLE. We note that there is no evidence that the MYH9 polymorphisms predispose to SLE itself (data not shown) based on genome-wide association data [17].

Among the smaller NIH lupus cohort, none of the seven MYH9 SNPs or E1 haplotype was independently associated with LN (data not shown). In addition, no evidence of E1 haplotype association was detected within the NIH cohort when contrasting the $8 \mathrm{LN}$ patients with serum creatinine concentrations $\geq 2.5 \mathrm{mg} / \mathrm{dl}$ versus the 52 lupus patients with serum creatinine values $<2.5 \mathrm{mg} /$ $\mathrm{dl}(\mathrm{p}=0.98$, additive model). Table 6 contains the results of the MYH9 E1 haplotype association analysis in all LN cases and SLE non-nephropathy controls from the combined PROFILE and NIDDK samples. No evidence of association was observed in these well-phenotyped cases and controls, nor was association detected for any individual SNP (ancestry-adjustment was not performed due to the lack of AIMs in the NIH samples).

We next tested whether differences in genotype frequencies were present between the Wake Forest SLEESRD cases $(n=25)$ and PROFILE ESRD cases $(n=31)$ with LN-associated ESRD. Significant differences in 
Table 5. Haplotypes of MYH9 with association of LN in PROFILE: $300 \mathrm{LN}$ cases vs. 246 SLE non-nephropathy controls

\begin{tabular}{llllllll}
\hline \multirow{2}{*}{$\begin{array}{l}\text { E1 } \\
\text { haplotype }\end{array}$} & \multirow{2}{*}{ Model } & \multicolumn{2}{l}{ Haplotype frequency } & & \multicolumn{2}{l}{ Recessive test } \\
& & cases & controls & & OR & $95 \%$ CI & p value \\
\hline \multirow{2}{*}{ GCCT } & dominant & 0.56 & 0.58 & & 1.08 & $0.68,1.70$ & 0.7552 \\
& additive & & & 0.92 & $0.71,1.18$ & 0.5079 \\
& recessive & & & 0.79 & $0.55,1.15$ & 0.2187 \\
\hline
\end{tabular}

\begin{tabular}{llllllll}
\hline \multirow{2}{*}{$\begin{array}{l}\text { E1 } \\
\text { haplotype }\end{array}$} & \multirow{2}{*}{ Model } & & \multicolumn{2}{l}{ Haplotype frequency } & & \multicolumn{2}{l}{ Recessive test } \\
& & cases & controls & & OR & $95 \%$ CI & p value \\
\hline \multirow{2}{*}{ GCCT } & dominant & 0.56 & 0.58 & & 0.99 & $0.67,1.48$ & 0.9763 \\
& additive & & & 0.93 & $0.75,1.17$ & 0.5389 \\
& recessive & & & 0.86 & $0.62,1.20$ & 0.3705 \\
\hline
\end{tabular}

Table 6. Haplotypes of MYH9 with association of LN in combined PROFILE and NIH samples: 357 LN cases vs. 286 SLE non-nephropathy controls with coincident SLE or whether MYH9 is associated with classic histologic changes of LN. We suspect the former scenario, based on the follow-up PROFILE and NIH results.

The different association results in subjects recruited in PROFILE, NIH and at Wake Forest may reflect the different sources of referral and phenotyping criteria and the lack of an SLE non-nephropathy group at Wake Forest. PROFILE and the NIH cohort were directed by rheumatologists, while Wake Forest recruitment was performed in dialysis facilities. Although ancestry data was not available in NIH samples, it is unlikely that differences in ancestry had a marked effect on these results based on the principal component-adjusted PROFILE data. It is apparent that cases with severe LN in PROFILE differed from those on dialysis and recruited at WFUSM. Contrasting genotypes between the WFUSM ESRD cases and PROFILE ESRD cases revealed that the E1 haplotype was present significantly more often in WFUSM cases $(p=0.037)$. Another possible explanation for these differences are population genetic substructure differences among study centers or geographic differences in environmental factors interacting with MYH9 kidney risk alleles.

Regarding issues of multiple testing, the primary inference based on the literature is the contrast of the homozygotes for the MYH9 E1 risk haplotype versus all other haplotypes (i.e. recessive model). For this strong a priori comparison, we made no multiple comparisons adjustment. For those SNPs within the E1 haplotype, the same philosophy holds. However, it is reasonable to make 
an adjustment for the three SNPs outside the E1 haplotype, even though there is strong linkage disequilibrium among these SNPs. We reference a Bonferroni adjusted p-value threshold relevant to those 3 SNPs (i.e. $0.017=$ $0.05 / 3$ ) and note that these SNPs were not associated in the PROFILE cohort even without adjustment.

Genetic association studies in LN have revealed association with kallikrein $K L K 1$ and $K L K 3$ promoter genes [19], the Fc receptor gene $F c \gamma R I I I a[20,21]$, tumor necrosis factor- $\alpha$-induced protein 3 TNFAIP3 [22], and the C1q genomic region in minority populations [23]. These gene families involve immune function and vascular reactivity. It is felt likely that MYH9 affects cytoskeletal integrity of the renal podocyte, although glomerular capillary thrombotic effects remain possible as abnormal platelets are present in MYH9-associated hematologic diseases (May-Hegglin anomaly, Fechtner, Sebastian and Epstein syndromes) [24]. MYH9 appears necessary for embryonic development, as heterocrosses of mice with targeted gene disruptions in MYH9 did not yield homozygotes [25] However, we feel that MYH9 does not play a major role in susceptibility to LN in AA.

In conclusion, this association analysis between $\mathrm{LN}$ and the MYH9 gene in AA fails to support a major role for MYH9 in causation of LN. The results support the need for replication analyses whenever genetic associa- tion is detected and suggests that the clinical diagnoses that are listed in the USRDS registry may not always be correct. A subset of AA with SLE and ESRD may have primary FSGS or FGGS (MYH9-associated nephropathies) with coincident SLE.

\section{Acknowledgements}

This work was supported in part by NIH grants RO1 DK070941 (B.I.F.), RO1 DK084149 (B.I.F.), RO1 DK53591 (D.W.B.), P01 AR49084 Program Project in the Genetics of Systemic Lupus Erythematosus, 1RC2 AR058951-01 National Consortia to Explore the Genotypic Basis for ESRD in Lupus and the NIDDK, NIAMS and NCI Intramural Research Programs. This project has been funded in whole or in part with federal funds from the National Cancer Institute, National Institutes of Health, under contract N01-CO-12400 and HHSN261200800001E. The content of this publication does not necessarily reflect the views or policies of the Department of Health and Human Services, nor does mention of trade names, commercial products, or organizations imply endorsement by the US Government. This research was supported in part by the Intramural Research Programs of the NIH, National Cancer Institute, Center for Cancer Research. The authors report no conflicts of interest in this work. The authors are indebted to the local nephrology community, including all physicians and their patients who participated, as well as to our study coordinators Joyce Byers, Carrie Smith, Mitzie Spainhour, Cassandra Bethea and Sharon Warren.

\section{References}

1 Kopp JB, Smith MW, Nelson GW, et al: MYH9 is a major-effect risk gene for focal segmental glomerulosclerosis. Nat Genet 2008;40:1175-1184.

- 2 Kao WH, Klag MJ, Meoni LA, et al: MYH9 is associated with non-diabetic end-stage renal disease in African Americans. Nat Genet 2008;40:1185-1192.

- 3 Freedman BI, Hicks PJ, Bostrom MA, et al: Polymorphisms in the non-muscle myosin heavy chain 9 gene (MYH9) are strongly associated with end-stage renal disease historically attributed to hypertension in African Americans. Kidney Int 2009;75:736-745.

-4 Freedman BI, Hicks PJ, et al: Non-muscle myosin heavy chain 9 gene $\mathrm{MYH} 9$ associations in African Americans with clinically diagnosed type 2 diabetes mellitus-associated ESRD. Nephrol Dial Transplant 2009;11: 3366-3371.

5 Fessel WJ: Systemic lupus erythematosus in the community. Incidence, prevalence, outcome, and first symptoms; the high prevalence in black women. Arch Intern Med 1974; 134:1027-1035.
6 Dooley MA, Hogan S, Jennette C, Falk R: Cyclophosphamide therapy for lupus nephritis: poor renal survival in black Americans. Kidney Int 1997;51:1188-1195.

7 Freedman BI, Wilson CH, Spray BJ, Tuttle $\mathrm{AB}$, et al: Familial clustering of end-stage renal disease in blacks with lupus nephritis. Am J Kidney Dis 1997;29:729-732.

$\checkmark 8$ Freedman BI: End-stage renal failure in African Americans: insights in kidney disease susceptibility. Nephrol Dial Transplant 2002;17:198-200.

-9 Freedman BI, Sedor JR: Hypertension-associated kidney disease: perhaps no more. J Am Soc Nephrol 2008;19:2047-2051.

10 Alarcon GS, McGwin G Jr, Petri M, et al: Baseline characteristics of a multiethnic lupus cohort: PROFILE 15. Lupus 2002;11:95101.

-11 Wigginton JE, Cutler DJ, Abecasis GR: A note on exact tests of Hardy-Weinberg equilibrium. Am J Hum Genet 2005;76:887-893.

12 Barrett JC, Fry B, Maller J, Daly MJ: Haploview: analysis and visualization of $L D$ and haplotype maps. Bioinformatics 2005;21: 263-265.
13 Tang H, Peng J, Wang P, Risch NJ: Estimation of individual admixture: analytical and study design considerations. Genet Epidemiol 2005;28:289-301.

14 Keene KL, Mychaleckyj JC, Smith SG, et al: Association of the distal region of the ectonucleotide pyrophosphatase/phosphodiesterase- 1 gene with type 2 diabetes in an African-American population enriched for nephropathy. Diabetes 2008;57:1057-1062.

15 US Renal Data System, USRDS 2008 Annual Data Report: Atlas of Chronic Kidney Disease and End-Stage Renal Disease in the United States. Bethesda, NIH, National Institute of Diabetes and Digestive and Kidney Diseases, 2009.

-16 Zarif L, Covic A, Iyengar S, Sehgal AR, Sedor JR, Schelling JR: Inaccuracy of clinical phenotyping parameters for hypertensive nephrosclerosis. Nephrol Dial Transplant 2000; 15:1801-1807. 
- 17 Harley JB, Arcon-Riquelme ME, Criswell LA, et al: Genome-wide association scan in women with systemic lupus erythematosus identifies susceptibility variants in ITGAM, PXK, KIAA1542 and other loci. Nat Genet 2008;40:204-210.

-18 Reeves-Daniel AM, Iskandar SS, Bowden DW, et al: Collapsing C1q nephropathy: another $\mathrm{MYH} 9$-associated kidney disease? Am J Kidney Dis 2010;55:e21-e24.

-19 Liu K, Li QZ, Gado-Vega AM, et al: Kallikrein genes are associated with lupus and glomerular basement membrane-specific antibody-induced nephritis in mice and humans. J Clin Invest 2009;119:911-923.
20 Salmon JE, Millard S, Schachter LA, et al: Fc $\gamma$ RIIA alleles are heritable risk factors for lupus nephritis in African Americans. J Clin Invest 1996;97:1348-1354.

21 Alarcon GS, McGwin G Jr, Petri M, et al: Time to renal disease and end-stage renal disease in PROFILE: a multiethnic lupus cohort. PLoS Med 2006;3:e396.

22 Bates JS, Lessard CJ, Leon JM, et al: Metaanalysis and imputation identifies a $109-\mathrm{kb}$ risk haplotype spanning TNFAIP3 associated with lupus nephritis and hematologic manifestations. Genes Immun 2009;10:470477.
23 Namjou B, Gray-McGuire C, Sestak AL, et al: Evaluation of $\mathrm{Clq}$ genomic region in minority racial groups of lupus. Genes Immun 2009; 10:517-524

24 Althaus K, Greinacher A: MYH9-related platelet disorders. Semin Thromb Hemost 2009;35:189-203.

25 Matsushita T, Hayashi H, Kunishima S, et al: Targeted disruption of mouse ortholog of the human MYH9 responsible for macrothrombocytopenia with different organ involvement: hematological, nephrological, and otological studies of heterozygous $\mathrm{KO}$ mice. Biochem Biophys Res Commun 2004; 325:1163-1171. 